\title{
Further Investigation of the Tolerance and Mechanical Adjustability of the *Acri.Tec AR-1 PC/IOL in Rabbit Eyes: An Intraocular Lens with Reversibly Adjustable Optical Power
}

\author{
Claus E. Jahn ${ }^{a}$ Ingeborg Fromberg ${ }^{b}$ Nicole Fesser $^{b}$ Ingrid Allgoewer ${ }^{b}$ \\ Christoph H. Jahn ${ }^{c}$ Nina S. Wakili ${ }^{c}$ Carmen R. Hofmann-Rummelt ${ }^{c}$ \\ Leonard M. Holbach ${ }^{c}$ Bernd A. Kamppeter ${ }^{d}$ \\ ${ }^{a}$ Augenpraxisklinik Dr. Jahn, Kempten, ${ }^{b}$ Acri.Tec GmbH, Glienicke, ' Universitätsaugenklinik Erlangen, Erlangen, \\ and ${ }^{d}$ Klinikum Mannheim der Medizinischen Fakultät der Universität Heidelberg, Mannheim, Germany
}

\section{Key Words}

Cataract surgery $\cdot$ Intraocular lens implantation $\cdot$ Refractive lens surgery $\cdot$ Adjustable intraocular lens $\cdot$ Animal study

\begin{abstract}
Purpose: To examine the tolerance and mechanical function of an adjustable intraocular lens (IOL) in rabbit eyes. Methods: Implantation of the *Acri.Tec AR-1 PC/IOL into 14 rabbit eyes. Manipulation of the lens 8 weeks after implantation in order to change the refractive power. Follow-up for up to 5 months. Histopathologic examination of the eyes. Results: Implantation and mechanical adjustment of the PC/IOL were possible. Eyes healed normally. No difference between eyes containing the *Acri.Tec AR-1 PC/IOL and eyes containing the control PC/IOL could be detected with respect to signs of inflammatory reaction, corneal transparency, intraocular pressure and histopathologic appearance. Histopathologic examination of the eyes showed that the *Acri.Tec AR-1 PC/ IOL did not cause any damage in rabbit eyes. Conclusion: The *Acri.Tec AR-1 PC/IOL is well tolerated in rabbit eyes for
\end{abstract}

The study was sponsored by *Acri.Tec GmbH, Henningsdorf, Germany. C.E.J. holds financial interest in this lens as a patent holder. (c) 2006 S. Karger AG, Basel

$0030-3747 / 06 / 0385-0309 \$ 23.50 / 0$

Fax +41613061234 E-Mail karger@karger.ch www.karger.com
Accessible online at:

www.karger.com/ore extended periods of time, suggesting that this $\mathrm{PC} / \mathrm{IOL}$ should be well tolerated in the long run. Surgical adjustment of the adjusting element can be performed with little effort several weeks after implantation.

Copyright $\odot 2006$ S. Karger AG, Basel

\section{Introduction}

Using ultrasound or optical biometry, there exists an inherent and unpredictable spread of postoperative refractions around the preoperatively determined target value which cannot be overcome [1-3]. Recently we reported results of the implantation of a reversibly adjustable intraocular lens (*Acri.Tec AR-1 PC/IOL) into rabbit eyes [4]. This PC/IOL was designed in order to improve postoperative refractive results in the surgical treatment of cataract. Also, this PC/IOL could potentially be helpful in cases in which cataract development in both eyes runs a different time course in the presence of pre-existing high ametropia or in eyes undergoing cataract surgery after laser in situ keratomileusis. It is also hoped that this $\mathrm{PC} / \mathrm{IOL}$ will be useful in pediatric cataract surgery, where increasing myopia develops as the child's eye grows over time. Other authors have recently proposed different

Claus E. Jahn, MD

Augenpraxisklinik Dr. Jahn

Bodmanstrasse 8

DE-87435 Kempten (Germany)

Tel. +49 831 14041, Fax +49 831 27294, E-Mail praxisdr.jahn@t-online.de 


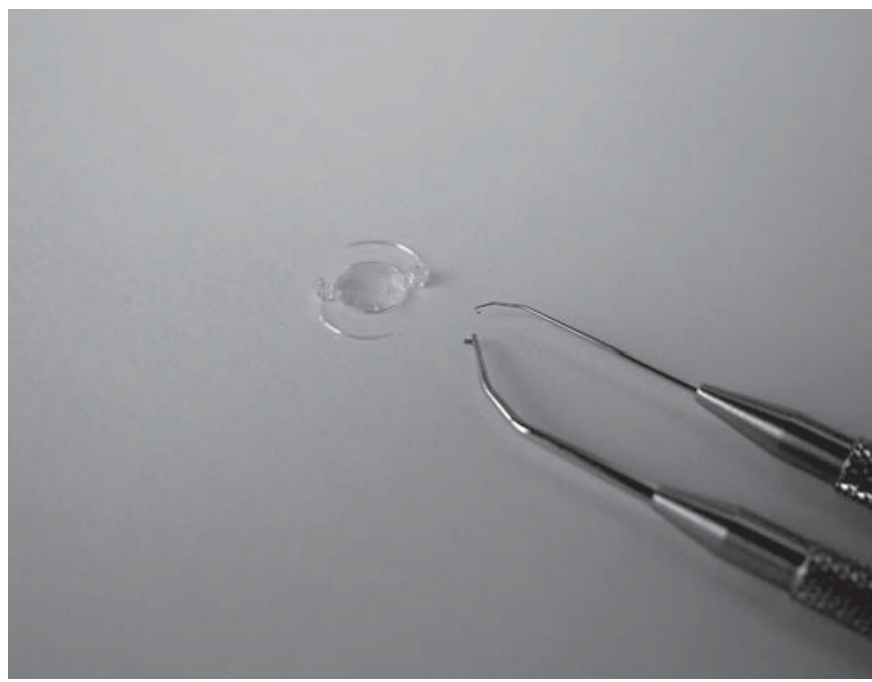

Fig. 1. *Acri.Tec AR-1 PC/IOL and instruments used for adjustment.

types of PC/IOLs which also aim at adjusting the refraction after the PC/IOL has been implanted into the eye [5-9].

In this study we further investigated the tolerance of the *Acri.Tec AR-1 PC/IOL after implantation into rabbit eyes. We also used this study for further refinement of the surgical approach for adjustment of the PC/IOL.

\section{Materials and Methods}

The prototype of the *Acri.Tec AR-1 PC/IOL is made of polymethylmethacrylate (PMMA; fig. 1). It is composed of an optical lens body of the usual characteristics, $5 \mathrm{~mm}$ in diameter. Haptics containing an adjustment element are connected to this body. This element is formed by a cylinder attached to the inner part of the haptic, which contains a piston attached to the outer part of the haptic. The cylinder and the piston can be moved relative to each other. This element allows the optical part of the PC/IOL to move along the optical axis of the eye. Adjustment of the lens is performed by means of two specifically designed hooks or forceps, and in humans would create a change of refraction of $1.5 \mathrm{dpt}$ per millimeter displacement (fig. 1). During adjustment surgery, the surgeon will apply pressure on the piston while applying a counterforce to the optic, e.g. by pressing onto the piston from above and holding a second instrument below the junction of the optic to the cylinder. Thus the piston will slide within the cylinder. The length of the displacement is controlled by the length of the hook pressing onto the piston. Since the haptic arms rest in a plane with a definite distance to the cornea and fovea, the movement creates a displacement of the optic along the optical axis.

As control PC/IOL, an *Acri.Tec 11 C PC/IOL made of PMMA with an optical diameter of $6 \mathrm{~mm}$ was implanted.
After obtaining consent from the Regierungspräsidium Karlsruhe, implantation and adjustment surgery was also carried out at the animal facility of the Faculty for Clinical Medicine Mannheim, University of Heidelberg. Sixteen white New Zealand rabbits, 6 weeks of age, were operated on under general anesthesia. In 14 animals an *Acri.Tec AR-1 PC/IOL was implanted into one eye, and the control eye received a conventional *Acri.Tec 11 C PC/IOL after phacoemulsification of the lens through a scleral tunnel. The posterior capsule was left intact. Four eyes of 2 rabbits were left aphakic. Intraocular pressure was also monitored by means of a Tono-pen.

Adjustment surgery was carried out in 4 rabbits after 2 months. We inspected the position of the components of the adjusting element through the operating microscope immediately after adjustment surgery. Postoperative observation took place for up to 5 months in unadjusted eyes and for up to 3 months in adjusted eyes. In both groups the process of capsular shrinkage continued with increasing length of the postoperative period and caused iris catch as well as secondary glaucoma in some eyes. As a consequence of this, 4 rabbits had to be sacrificed before the time of 5 months after implantation. This was due to secondary glaucoma affecting 2 rabbits with glaucoma in the control eye and 2 rabbits with glaucoma in the eye containing an *Acri.Tec AR-1 PC/IOL. After 5 months the remaining 12 rabbits were sacrificed.

All eyes were examined histopathologically using standard techniques of light microscopy. Endothelial cell density was counted as cells per viewing field at $400 \times$ magnification. Amounts of fibrin and white blood cells were judged by a scale of 5 classes expressed from 0 (absent) to 4 (abundant). Descriptive statistical results were expressed as means \pm standard deviation for continuous variables or as median and range for discrete variables.

\section{Results}

The *Acri.Tec AR-1 PC/IOL was easily implanted into the lens capsule of rabbit eyes after removal of the lens by phacoemulsification.

The *Acri.Tec AR-1 PC/IOL fitted into the capsule and centered well there. The eyes healed just like the control eyes and were macroscopically free of inflammation 4 weeks after surgery and thereafter. Intraocular pressure was below $20 \mathrm{~mm} \mathrm{Hg}$ in most of the eyes containing the *Acri.Tec AR-1 PC/IOL as well as in the eyes containing the control IOL. In both groups many eyes developed a forward movement of the PC/IOL due to massive lenticular regeneration and capsular shrinkage in the following months. This occurs commonly in the rabbit eye and led to the formation of anterior and posterior synechiae in some eyes of both groups. In 2 eyes of each group, this was associated with secondary glaucoma.

Adjustment of the *Acri.Tec AR-1 PC/IOL was carried out in 4 eyes after 2 months. The displacement of the adjusting element was demonstrated immediately after sur- 
gery by inspection through the surgical microscope. The adjusted eyes healed in a normal fashion.

Gross pathologic examination revealed that the *Acri. Tec AR-1 PC/IOL was well centered inside the lens capsule. The posterior capsule and all zonules were intact. Histopathologic examination of the eyes did not show damage to the cornea, iris, ciliary body or the retina or signs of inflammation in any of the eyes without glaucoma (table 1). By contrast all eyes demonstrated massive regeneration of lens epithelial cells, with subsequent metaplasia of epithelial cells into bladder cells. This created an enormous volume of cells and cell debris, which in some eyes extended through the pupil into the anterior chamber.

\section{Discussion}

Previously we reported the successful manufacturing and implantation into rabbit eyes of a new type of $\mathrm{PC} / \mathrm{IOL}$ ( ${ }^{*}$ Acri.Tec AR-1 PC/IOL), which has an adjusting element so that the position of the lens optic can be moved, during a second operation, along the optical axis of the eye after implantation of the PC/IOL. In this way it should be possible to move the focus of the pseudophakic eye and place it exactly on the fovea for the focal distance of the patient's personal preference [4]. In theory this adjustment might be altered several times, if an unsatisfactory refraction was achieved by lens implantation or if the patient wished so because of a change in lifestyle. This first study demonstrated the overall validity of the technical approach as well as the short-term tolerance of the ${ }^{*}$ Acri.Tec AR-1 PC/IOL. In the present study we aimed to obtain further evidence for the extended tolerance of this type of IOL.

The results of this study confirm that implantation of the *Acri.Tec AR-1 PC/IOL is technically feasible. Healing of the eyes ran a normal course comparable to healing after implantation of a conventional PMMA PC/IOL. After healing the eyes containing the *Acri.Tec AR-1 PC/ IOL remained free of intraocular or extraocular inflammation during the follow-up period, when compared to the conventional PMMA IOL. Corneal transparency was not reduced in either group so that corneal damage is not to be expected from the implantation of the ${ }^{*}$ Acri.Tec AR-1 PC/IOL into human eyes. There was no difference between the number of eyes in the two pseudophakic groups with regard to the development of secondary glaucoma as a consequence of synechia formation, which is a distinct phenomenon of the eyes of young rabbits as were
Table 1. Histopathologic findings in eyes containing the *Acri.Tec AR-1 PC/IOL (AR IOL) and control PC/IOL

\begin{tabular}{lcc}
\hline & AR IOL & Control IOL \\
\hline Mean corneal endothelial count & $29 \pm 4$ & $28 \pm 4$ \\
Median fibrin intensity 0-3 & $2(1-3)$ & $2(1-3)$ \\
Median lymphocyte count & $1(1-3)$ & $1(1-3)$ \\
Median granulocyte count & $1(1-3)$ & $1(1-3)$ \\
\hline Eyes with open angle, \% & 100 & 100 \\
Eyes with normal iris, \% & 100 & 100 \\
Eyes with normal ciliary body, \% & 100 & 100 \\
Eyes with normal retina, \% & 100 & 100 \\
\hline
\end{tabular}

Figures in parentheses indicate ranges.

used in this experiment, and has no relevance to the situation encountered in surgery for senile cataract.

Centering and adjustment of the *Acri.Tec AR-1 PC/ IOL remained stable. No tilting occurred during the time of clinical monitoring. Adjustment of the *Acri.Tec AR-1 PC/IOL 8 weeks after implantation was carried out bimanually through two corneal paracenteses $0.9 \mathrm{~mm}$ wide. During adjustment surgery we also demonstrated that there is substantial room behind the iris and in front of the optic of a PC/IOL which can accommodate an adjustment device extending parallel to the optical axis. In humans this is to be expected, since the natural lens has a thickness of about $5 \mathrm{~mm}$ centrally while the thickness of a PC/IOL made of PMMA is around $0.2 \mathrm{~mm}$. In fact one can regularly demonstrate the existence of a gap between the iris and an implant upon slitlamp biomicroscopy. Healing after adjustment surgery was quick and uneventful, indicating that this type of surgery will not be harmful.

There are other types of adjustable IOLs under development which either change the refraction by light-induced modifications of the IOL material or by moving the optic along the optical axis [6-9], demonstrating that there are several possible approaches to the problem of postimplantation refractive adjustment.

In summary the results from this study suggest that the mechanical concept of this adjustable $\mathrm{PC} / \mathrm{IOL}$ is valid and that implantation of the *Acri.Tec AR-1 PC/IOL into human eyes would be feasible and safe. 


\section{References}

1 Olson T, Andersen CU, Plesner HJ: Computerized intraocular lens calculation: clinical results and predictability. Br J Ophthalmol 1989;73:220-224.

2 Hoffer KJ: Clinical results using the Holladay 2 intraocular lens power formula. J Cataract Refract Surg 2000;26:1233-1237.

3 Kijima T, Kozawa T, Kora Y, Yaguci S, Inatomi M, Koide R, Ozawa T: Accuracy of intraocular power calculation formulas. Nippon Ganka Gakkai Zasshi 1999;103:470476 .
4 Jahn CE, Jahn MA, Kreiner CF, Serester A, Fromberg I, Jacobi S, Deutschmann S, Schäffer EH, Kamppeter BA: Intraocular lens with reversibly adjustable optical power: pilot study of concept and safety. J Cataract Refract Surg 2003;29:1795-1799.

5 Werblin TP: Multicomponent intraocular lens. J Cataract Refract Surg 1996;12:187189.

6 Schwartz DM, Jethmalani JM, Sandstedt CA, Kornfield JA, Grubbs RH: Postimplantation adjustable intraocular lenses. Opthalmol Clin North Am 2001;14:339-345.
7 Matthews MW, Egglestone HC, Hilmas GE: Development of a repeatedly adjustable intraocular lens. J Cataract Refract Surg 2003; 29:2204-2210.

8 Matthews MW, Egglestone HC, Pekarek SD, Hilmas GE: Magnetically adjustable intraocular lens. J Cataract Refract Surg 2003;29: 2211-2216.

9 Schwartz DM: Light-adjustable lens. Trans Am Ophthalmol Soc 2003;101:417-436. 